\title{
INTRODUZIONE GENERALE
}

\author{
PIERCARLO NICOLA $(*)$ \\ ANGELO ZANELLA $(* *)$
}

E' presente a tutti l'importanza, in ambito nazionale, della misurazione del cosiddetto Prodotto Interno Lordo e del tasso di inflazione quali indicatori della situazione economica complessiva, ma è anche comune esperienza come sia difficile ottenere delle previsioni, con le relative implicazioni per la realtà economica futura, che possano ritenersi veramente attendibili, a differenza, ad esempio, della conoscenza delle grandezze fisiche, le cui implicazioni reali, nei casi correnti, sono note e sicure.

L'incontro esamina le possibilità offerte dai metodi matematici e statistici, nella formulazione di previsioni e decisioni economiche, con lo sguardo, da un lato, a quanto è possibile e corrente fare con le tecnologie collegate alle scienze naturali, ma dall'altro, al riferimento, generalmente non deterministico, costituito dalle scelte degli operatori economici essenziali, quali sono i consumatori e le imprese.

La giornata di studio si apre con una riflessione in termini generali sulla relazione fra scienza economica e scienze naturali e conseguenti tecnologie; segue la presentazione delle idee fondamentali di Vilfredo Pareto e della sua convinzione dell'esistenza di una stretta analogia fra Economia teorica e Meccanica razionale; vengono, poi, presentate le reali possibilità predittive insite nell'utilizzazione dell'Economia

(*) Istituto Lombardo Accademia di Scienze e Lettere, Università degli Studi di Milano.

${ }^{(* *)}$ Istituto Lombardo Accademia di Scienze e Lettere, Università Cattolica del Sacro Cuore di Milano. 
matematica e dell'Econometria. La dicotomia fra scienze naturali e scienze umane porta in corrispondenza a sottolineare come le scienze naturali si basino su misure di grandezze ottenute mediante procedimenti oggettivi di confronto, diretto o indiretto, con una unità di misura - riproducibili nelle stesse condizioni - mentre per le scienze umane, come l'Economia, invece è tipico il ricorso a valutazioni - spesso ricondotte, attraverso la nozione di "utilità", a valori monetari - dove, però, è comunque presente una componente soggettiva e psicologica. Alberto Quadrio Curzio, nel contributo Prospettive e limiti dell'Economia Quantitativa, discute aspetti metodologici della Teoria economica con gli obiettivi della previsione e del controllo. La relazione sottolinea in modo particolare la contrapposizione fra Economia reale ed Economia finanziaria. L'Economia reale si riferisce alla produzione, allo scambio ed al consumo dei beni, nel cui ambito la capacità di previsione - almeno nel breve periodo - è generalmente ritenuta accettabile, mentre l' Economia finanziaria si riferisce agli strumenti introdotti negli ultimi trenta anni al fine di agevolare la formazione del risparmio e del suo impiego per gli investimenti. L'Autore pone l'accento sul fatto che, in quest'ambito, invece, la capacità di previsione è tuttora molto debole.

Luigi Pasinetti, nella relazione con lo stesso titolo, Prospettive e limiti dell'Economia Quantitativa, sviluppa autonomamente il medesimo tema. Egli riconosce l'importanza dello formulazione matematica, ma mette in guardia contro i pericoli connessi all'inevitabile astrattezza della formulazione matematica, la quale rischia di allontanare le conclusioni dalla realtà economica che intende rappresentare e prevedere. L'Autore sottolinea con forza l'essenzialità della riflessione profonda sulla corrispondenza alla realtà economica delle ipotesi fatte, che vincolano la validità concreta dei risultati matematici.

Pier Carlo Nicola, nella relazione Economia matematica e meccanica razionale, mette in evidenza l'importanza della nozione di equilibrio generale di estrazione Walrasiano - Paretiana chiaramente collegata alla Meccanica Razionale. L'Autore non manca, tuttavia, di sottolineare come in Marshall l'analogia biologica sia presentata quale forse più promettente, ma certo anche più complessa. Egli osserva, infine, come i modelli di equilibrio generale male si prestino alla previsione.

Giovanni Marseguerra, nel contributo La formalizzazione matematica nelle teorie economiche: problemi e prospettive, si sofferma sui rapporti fra modelli teorici e conoscenza economica, sottolineando con 
vigore la necessità, per quest'ultima, di molteplici collegamenti con altre discipline, quali la Sociologia e la Psicologia. Inoltre l'Autore fa presente l'importanza dei modelli di simulazione di sistemi economici mediante computer, sempre più accentuata vista la loro larga diffusione. Marseguerra indica come particolarmente promettenti quelli facenti capo alla cosiddetta Agent-based Computational Economics che, attraverso la simulazione dei comportamenti ripetuti di agenti autonomi operanti in ambiti complessi, cerca di comprendere la formazione di talune regolarità nell'andamento temporale dei fenomeni economici. Si tratta di una recente e moderna evoluzione nell'utilizzo del computer in economia che si differenzia significativamente rispetto alle tradizionali metodologie con le quali gli economisti, a partire da un modello teorico di risposta, cercano di valutare le conseguenze sull'andamento temporale di variazioni nei valori dei parametri del modello e delle condizioni iniziali.

Mario Faliva e Maria Grazia Zoia, nella relazione Econometria: istanze dell'Economia e parallelismi con le scienze naturali, propongono una interessante interrelazione fra Economia ed Econometria. Da un lato l'Economia teorica, con il ricorso agli strumenti matematici e statistici, suggerisce, in via naturale, i modelli di tipo econometrico, la cui validazione mediante l'utilizzo dei risultati sperimentali consente la conferma della teoria; d'altro lato l'analisi sperimentale delle serie storiche, condotta in base a modelli interpretativi econometrici, cioè, a relazioni matematiche e statistiche, accertata la coerenza di fondo degli stessi con i principi dell'economia teorica, può reciprocamente suggerire degli eventuali aggiustamenti o modifiche di un modello teorico di riferimento, che così risulta arricchito.

La relazione di Fausto Borgonovi, Procedimenti di misurazione, predicibilità e determinismo nelle leggi della Fisica, costituisce un allargamento del contesto, in quanto permette di farsi un'idea delle problematiche e degli approcci alla base della Fisica moderna e di stabilire possibili analogie con l'Economia quantitativa come pure eventuali spunti per nuove ricerche. Si fa presente come il fisico e l'economista matematico cerchino entrambi di fare delle previsioni, di struttura concettualmente simile, basandosi su modelli matematici, vale a dire, in sostanza, su equazioni tra variabili che possono essere osservabili, cioè misurabili a meno di un errore stimabile. Il ricorso alla Statistica probabilistica, che spesso viene indicato come espressione della teoria dell' $i$ gnoranza - utilizzato per supplire alla carenza di informazioni nei con- 
fronti di sistemi complessi - può, invece, rappresentare il metodo appropriato, come accadde per la Meccanica statistica che ha creato un ponte fra le leggi microscopiche della Meccanica, a cui sono soggetti i singoli atomi o le singole molecole, e le leggi della Termodinamica, che si riferiscono alle variabili macroscopiche - fra le quali figura la temperatura - e può forse consentire delle analogie nello studio dell'equilibrio economico generale. Senza possibilità, al momento attuale, di diretti confronti appare, invece, la meccanica quantistica con carattere intrinsecamente probabilistico e statistico.

Nella relazione di Angelo Zanella, Procedimenti di misurazione nelle discipline economico-sociali, si considera, come elemento di confronto, il procedimento di misura di una grandezza in ambito tecnologico nel quale è possibile accertare il valore vero della grandezza di interesse, a meno di un errore aleatorio trascurabile, e quindi acquisire, ad un certo istante, in modo sostanzialmente esatto, lo stato di funzionamento di un processo. Si nota, quindi, come per le grandezze economiche, frequentemente di natura monetaria, con una finalità concettualmente simile, si tratti di accertare, per un determinato periodo, l'ammontare totale o medio della caratteristica di una popolazione e si debbano utilizzare, per la loro stima, i risultati di indagini campionarie, talvolta anche complesse, che ne costituiscono l'aspetto peculiare: l'errore medio di misura risulta quindi incrementato, rimanendo però ancora stimabile, a causa della variabilità dovuta al campionamento e collegata a quella oggettiva della popolazione.

L'obiettivo diviene diverso se si è interessati, non solo ad accertare una situazione in un determinato periodo di tempo, ma si vogliono anche stabilire delle previsioni per periodi futuri in base ad un modello economico e/o econometrico. In questo caso l'errore di misura e di campionamento può risultare incrementato in modo non direttamente valutabile a causa della non esatta conoscenza del modello interpretativo. Un caso tipico viene ravvisato nella previsione della misura dell'inflazione.

Si osserva, infine, che la condizione di equilibrio di una economia di mercato può collegarsi alle scelte di individui che operano in accordo ad un'entità difficilmente definibile in termini generali e, pertanto, difficilmente misurabile, indicata come Utilità. Di fronte ad una pluralità di alternative soggette ad incertezza, che è una situazione tipica del "decisore" in ambito finanziario e d'impresa, la teoria di von Neumann-Morgenstern-Savage-de Finetti - che è stata soggetta a valu- 
tazioni critiche, revisioni e complementi - può essere utile per orientare verso le scelte che massimizzano l'utilità media, rispetto alle distribuzioni di probabilità ipotizzate, per valutare le possibili conseguenze delle decisioni. 